\title{
A NOVEL METHOD FOR DETECTING THE CHAOTIC BEHAVIOR IN DYNAMICAL SYSTEMS WITH APPLICATIONS ON CHUA'S SYSTEM
}

\author{
Abdel-Azeem Mohamed and Samy H. Darwish \\ Faculty of Engineering, Pharos University, Alexandria, Egypt \\ azima65@hotmail.com, and salord1960@yahoo.com
}

\begin{abstract}
The important step in studying the qualitative behavior of non-linear dynamical system is how to detect the presence of chaos. There are several methods that used to determines the presence of chaos signature. This paper presents a novel method in detecting the presence of chaos. The method combined two techniques namely: the normal form analysis and largest Lyapunov exponent (LLE). Computerized algebraic programs were generated to investigate these two techniques. An example was given to furnish the herein given computer algebra techniques based on real applications. The results obtained in this work were verified with results published by other researchers. The suggested method can provide highly active and efficient ability when studying the nature of non-linear dynamical systems and its chaotic presence.

Keywords - Chaos, Normal form, largest Lyapunov exponent.
\end{abstract}




\section{Introduction}

Many tools can be used to study the qualitative behavior of linear or non-linear dynamical systems. In general non-linear systems cannot be analyzed completely in a systematic manner. Therefore, some approaches and techniques can be used to represent the non-linear system as follows: describing function approach can be used when a transfer function of the linear part of the non-linear system is available; but Volterra series where used in the case of weakly non-linear systems, while harmonic balance can be used when single non-linearity exists. Other numerical techniques with advantages and disadvantages to analyze non-linear systems were developed in [1-4]. Many problems and limitations arise when using the above approaches and techniques. The biggest problem is how to find an algorithm that enables the solution of the non-linear dynamical system and keeps errors minimum; besides, it should also offer simplicity in use as well as quick convergence.

This paper focuses on two approaches for studying the qualitative behavior of the nonlinear system. The first one is the normal form theory [5-10] and the second is the LLE technique [11-14]. The normal forms are used to facilitate and help in diagnosing-the onset of chaos in non-linear systems while calculations of Lyapunov exponents are a way to study where a system is chaotic or not. In the speaking about Lyapunov exponents, the largest one is meant. A positive LLE indicates that the system is chaotic, while a negative one indicates the non-chaotic behavior. The LLE can also be used to analyze the stability of non-linear systems [15-18]. A new algorithm was created to satisfy the idea of the suggested methodology; as illustrated in fig.1. This algorithm gives some advantages as: fast convergence to steady state solutions, easy implementation, reliable for higher dimensional systems and low number of iterations required. Section 2 gives an outline of normal forms method for non-linear dynamical systems. The computation procedures for the LLE are presented in section 3. Section 4 illustrates the suggested methodology. While section 5 gives a comparative study with an applicable example to show the validity of the adopted algorithm.

\section{Normal forms for non-linear dynamical system}

Consider the autonomous non-linear system given by the following state equation:

$$
\frac{d x(t)}{d t}=A x(t)+B F_{n}(x(t))
$$

Where,

$A$ and $B$ are real matrices of constant coefficients and compatible dimensions, and $F_{n}(x(t))$ is the vector of non-linear functions that satisfy some regularity conditions such as smoothness, continuity, and should be totally Lipchitz.

The normal form theory depends upon successive coordinate transformations to construct a simple form and find a new system with a topologically that conjugate to the original system in a simple canonical form. However there are many techniques for finding the normal forms and most of these techniques lack generality. Hartman-Grobman method uses the eigenvalues of the linearized system. If one of the eigenvalues lies on the 
imaginary axis, the linearization fails, and the process is terminated. The Poincare' method does not work when there are resonance relation between the eigenvalues. Both Poincare' and Taken methods give the same result for vector fields having a diagonalizable linear part. Poincare' method not applied to non-diagonalizable vector fields. Ushiki normal form method which is the most suitable technique; is based on the Lie brackets and the K-jets [6],[7].

Ushiki method may be considered as a refinement of Takens' method. The advantage of this method is that it does not need an eigenvalue decomposition technique. In what follows the adaptation of the Ushiki method in the calculation of the normal form of nonlinear system described by equation (1) is presented. The $\mathrm{K}^{\text {th }}$ order normal form of the non-linear system in (1) can be obtained by solving the following equation:

$$
\frac{d}{d t} g_{k}(t)=\pi_{k}\left(\left[Y^{k-1}, V^{k-1}+g_{k}(t)\right]_{k}\right)
$$

With, $\left[Y^{k-1}, V^{k-1}\right]^{k-1}=0$

Where,

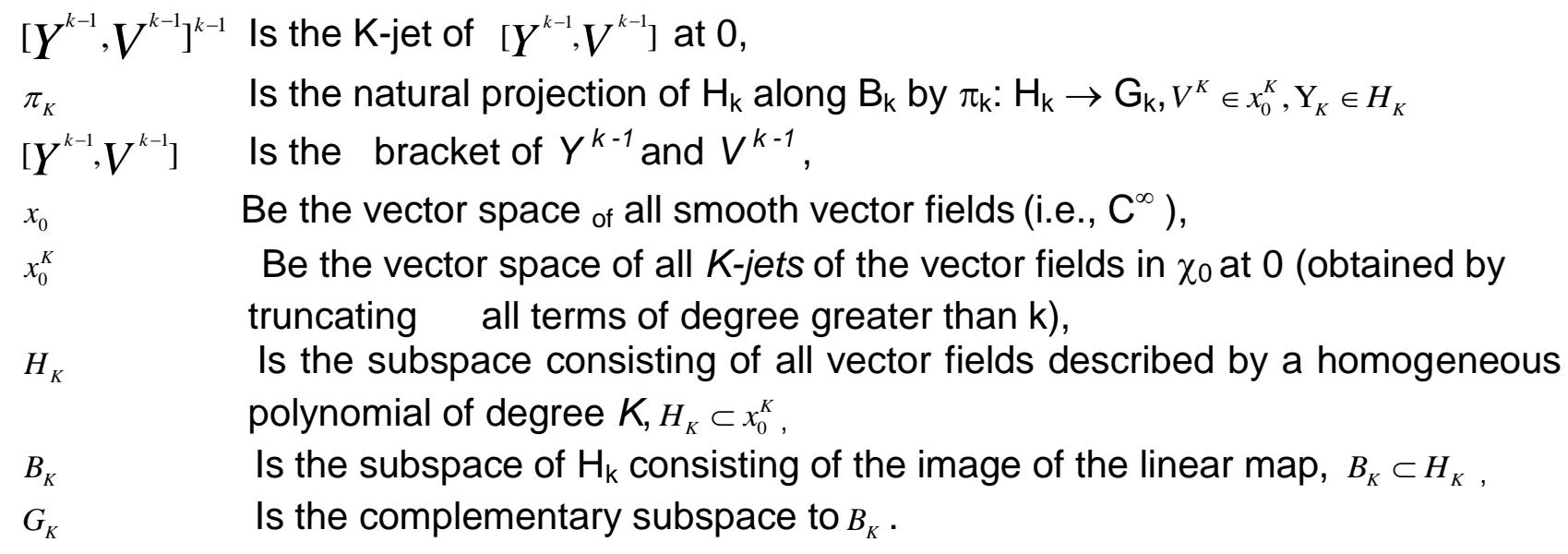

\section{Lyapunov exponents}

There is dependency between Lyapunov exponents and the chaotic properties of dynamical systems. There are several techniques for finding the Lyapunov exponents of the non-linear systems given by equation (1) [11-15]. However, no single technique appears to be optimal for calculating the Lyapunov exponents. For calculating the LLE depends upon the time domain solution of the state equation, some methods can be described as:

\subsection{The standard method}

This method is most popular method to compute the Lyapunov exponents that combines the numerical integration of the linear evaluation equation with the Gram-Schmidt reorthonormalization process applied periodically. To find the approximate exponents $\lambda_{i}(\mathrm{t})$ at time $\mathrm{t}=\tau$, the renormalization internal T was picked, so that $\tau=\mathrm{rT}$ with $\mathrm{r} \in \mathrm{N}$, the linear 
evaluation equation is integrated over $\mathrm{T}$ and the Gram-Schmidt process applied. This is repeated a total $r$ times keeping the norms from the Gram-Schmidet (GS). The GS ensures that the direction and rates of growth are measured correctly [11-15]. The Lyapunov exponents are:

$$
\lambda_{i}=\lim _{r \rightarrow \infty} \frac{\sum_{m=1}^{r} \log \left(N_{i}(m)\right)}{r T}
$$

Where:

$\mathrm{Ni}(\mathrm{m}) \quad$ is the norm of the distance between two trajectory points

$r \quad$ is the number of iterations,

$T \quad$ is the interval period of renormalization.

This method needs a good choice of $T$ and large number of iterations since a bad choice of $T$ may cause the algorithm to fail.

\subsection{The $Q-R$ algorithm}

It is another technique for finding the Lyapunov exponents. This method depends upon decomposition of the fundamental solution of the system. The fundamental solution is expressed as $M(t)=Q(t) R(t)$, where $Q(t)$ is an orthogonal matrix and $R(t)$ is an upper triangular matrix which are functions of both $Q(t)$ and the Jacobian matrix of the system (1) [11-15]. The Lyapunov exponents are obtained from the diagonal elements of the matrix $R(t)$ and are given by:

$$
\lambda_{i}=\lim _{t \rightarrow \infty} \frac{1}{t} \log R_{i i}(t), 1 \leq i \leq n
$$

This method needs accurate evaluation of the orthogonal matrix, since the error in orthogonality leads to a break down in the computations of Lyapunov exponents due to numerical over and under flows. The two methods require rescaling, reorthogonalization and large number of iterations.

A new algorithm based on combining the normal form theory and the numerical calculation of the LLE to satisfy the qualitative behavior of non-linear systems is illustrated in the next section.

\section{The suggested methodology:}

This method don't need rescaling or re-orthogonalization so it lends itself to fast computation, easy implementation and reliable for all systems of higher order. It also avoids numerical over flow and takes the advantage of making use of all available data in the time series solution of the differential equation. Fig. 1 illustrates the proposed algorithm procedures as follows: The normal form expressions obtained from the system of differential equations are used to convert the non-linear dynamical system to simple equivalent one. Time series data were generated from the equivalent system to evaluate LLE. Then the presence of chaos can be detected. 


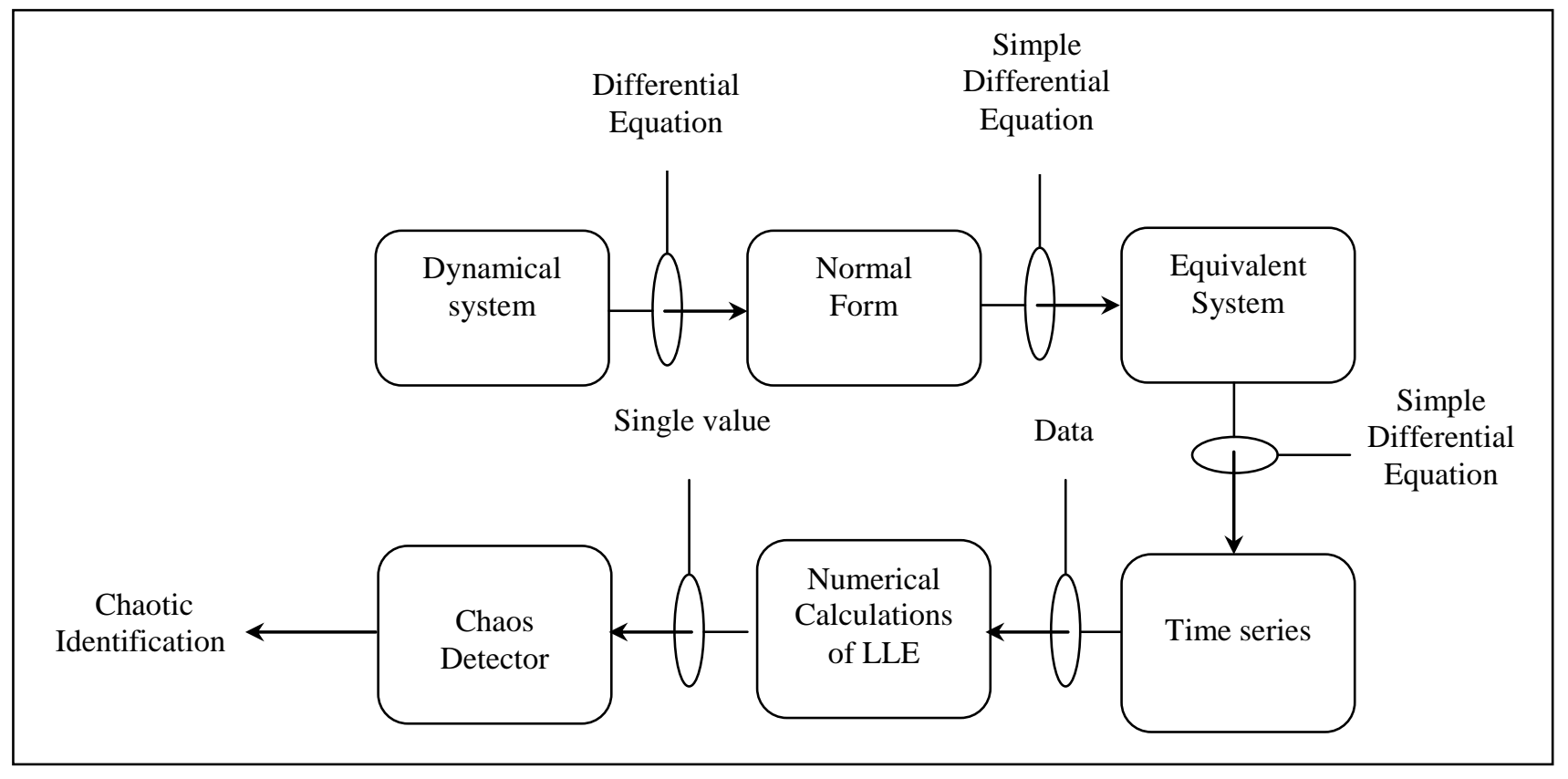

Fig. 1 Block diagram of the proposed algorithm

\section{The proposed approach proceeds as follows:-}

- Obtain the normal form expressions as given in (2).

-Use the normal form expressions to obtain the time domain solution with the advantage of fast conversion.

-Calculate the LLE from time domain solution.

The outcome of this algorithm is the Lyapunov exponents of the system, the largest of which $\lambda_{i}$ is of special interest in diagnosing dynamical systems, while zero values of $\lambda_{i}$ indicate periodic behavior. Negative values of $\lambda_{i}$ indicate stable fixed points, while positive values of $\lambda_{\mathrm{i}}$ indicate chaotic behavior and presence of chaos[12], [16], and [18]. The $i^{\text {th }}$ Lyapunov exponent is given by:

$$
\lambda_{i}=\frac{1}{N} \sum_{i=1}^{N} \log \left|\frac{d_{i}}{d_{o}}\right|
$$

Where:

$N \quad$ is the number of solution points obtained by direct integration.

$d_{o} \quad$ is the initial separation between two nearby points along the trajectory.

$d_{i} \quad$ is the $i^{\text {th }}$ distance between two nearby points on the trajectory.

Examples for attractors of a three dimensional system are given in table.1. 
Table.1. Examples of attractors of a three-dimensional system

\begin{tabular}{|c|c|c|}
\hline Attractor & Sign of Lyapunov exponents & Asymptotic dynamics \\
\hline Stable equilibrium & $(-,-,-)$ & Stationary \\
\hline Stable periodic orbit & $(0,-,-)$ & Periodic \\
\hline Attracting torus & $(0,0,-)$ & Quasi-periodic \\
\hline Strange attractor & $(+, 0,-)$ & Chaotic \\
\hline
\end{tabular}

The next section introduces some examples to illustrate the procedure. A Computer algebra program is designed using package (Maple) to find normal forms. The calculation of the LLE is carried out using an algorithm designed specially in $\mathrm{C}^{++}$to handle the computation task.

\section{5- Applicable example}

This example introduces a studying Chua's system as follows:

a) Comparison between the analytical solution as normal form and numerical computing one as Runge-Kutta-4 method.

b) Comparison between LLE calculated using previous methods ( $Q-R$ algorithm and standard method) and such calculated based on the proposed method.

\subsection{Chua's oscillator}

Chua's oscillator is one of the simplest electronic circuits that are capable of producing chaos. It can exhibit a wide array of behavior including a great variety of attractors, bifurcations, and routes to chaos[19].

\subsubsection{Chua's system representation}

The Chua's system with cubic nonlinearity is described by[20].

$$
\begin{aligned}
& \frac{d x}{d t}=\alpha\left(y+c x-x^{3}\right) \\
& \frac{d y}{d t}=x-y+z \\
& \frac{d z}{d t}=-\beta y
\end{aligned}
$$


Where, $\beta, \alpha$, and $c$ are system parameters. For the set of parameters $(\alpha=10, \beta=16$, and $c=0.143$ ) the system was shown to display Chaos (double scroll attractor) [20].

The local Lyapunov exponents can be calculated from the Jacobian matrix at equilibrium point $(x=y=z=0)$ which is given by:

$$
J=\left[\begin{array}{ccc}
-\alpha c & \alpha & 0 \\
1 & -1 & 1 \\
0 & -\beta & 0
\end{array}\right]
$$

The sum of the Lyapunov exponents is given by:

$$
\sum_{i=1}^{3} \lambda_{i}=\lim _{t \rightarrow \infty} \sup \frac{1}{t} \int_{0}^{t} \operatorname{Trace}(J(s)) d s
$$

The result of the calculation gives,

$\sum_{i=1}^{3} \lambda_{i}=-\alpha c-1=-2.43$

This result indicate that the system is dissipative

\subsubsection{Normal form of Chua's system}

For the Chua's system, the $3^{\text {rd }}$ order normal form is given by:

$$
v^{3}=\omega\left(x \frac{\partial}{\partial x}+y \frac{\partial}{\partial y}\right)+\delta\left(y \frac{\partial}{\partial x}-x \frac{\partial}{\partial y}\right)+\sigma z \frac{\partial}{\partial z}
$$

Where: $\omega, \delta$ and $\sigma$ are the arbitrary constants from the integration of the Ushiki normal form.

\subsection{The comparative analysis}

5.2.1 System behavior using normal form method:

To check the validity of the proposed algorithm, a further illustration of the phase plane and time domain solution using the normal form expressions were illustrated in figure (3): 


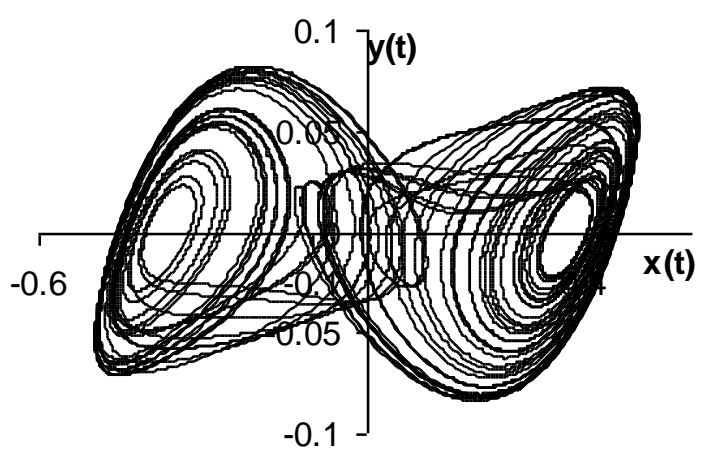

Fig.2 (a) The phase plan plot $(x(t), y(t))$ of Chua's system using Normal form method

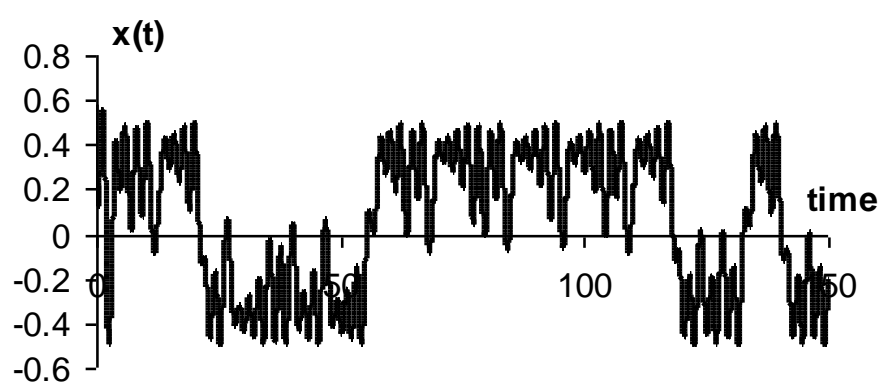

Fig.2 (b) The time response $x(t)$ of Chua's system using Normal form method.

\subsubsection{System behavior using Runge-Kutta-4 method:}

The numerical solution of the system using the Runge-Kutta-4 method [4] with step size $\mathrm{h}=0.01$ is shown in the phase plane plot and time domain solution of fig. 3 .

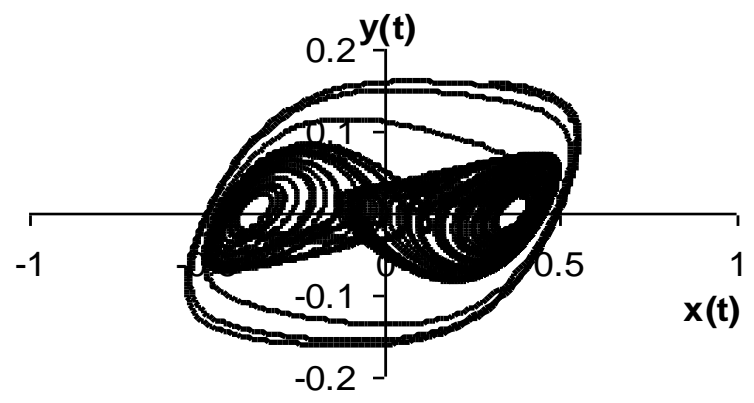

Fig. 3(a) The phase plane plot $(x(t), y(t))$ of Chua's system using Runge-Kutta-4 method 


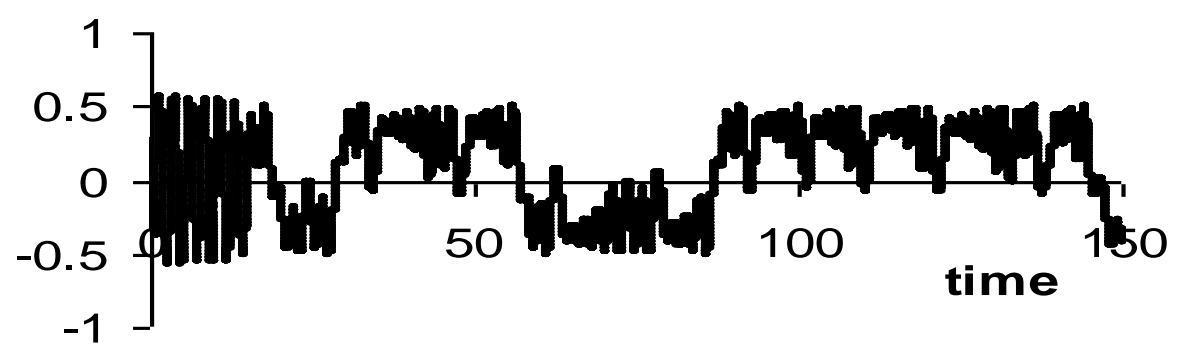

Fig. 3(b) The time response $x(t)$ of Chua's system using Runge-Kutta-4 method

Figures 2, 3 illustrate that the qualitative behavior of analytical solution is the same as that obtained by the numerical methods with the advantages of fast convergence to steady state behavior. However solutions give the same response, the solution satisfied by the normal form is free from transient that shown in fig.3 (b).

\subsubsection{LLE calculations for Chua's system}

The standard method and the Q-R algorithm are both used to calculate the LLE, N=10000 iterations as shown in table (2).

Table.2. Comparison between existing technique and the proposed approach

\begin{tabular}{|c|c|c|c|}
\hline Algorithm & Standard method & Q-R algorithm & Proposed approach \\
\hline LLE & 0.1332 & 0.1328 & 0.1317 \\
\hline Run time (sec) & 3.09 & 3.99 & 1.84 \\
\hline
\end{tabular}

From this table; the \% reduction in Run time about $49 \%$ to $59 \%$. The calculations give positive value of the LLE which indicates chaotic behavior (double scroll attractor) and is in agreement with the results in [12],[16],[20].

It is seen from table (2) that the calculated $\lambda_{I}$ as LLE by using the proposed approach is in good agreement with the existing algorithms with the advantage of minimum run time. 


\section{Conclusions}

- Many numerical techniques are used to investigate the qualitative behavior of the non-linear systems and computing the LLE.

- Most of the used methods have the disadvantages of lack of generality, less accuracy, difficult implementation, and numerical overflows/underflows for higher dimensional systems and give unreal behavior especially in chaotic systems.

- The proposed algorithm that combined the normal form method and the numerical method can provide active solutions to many challenges, avoids most computational errors encountered in other numerical methods, besides it is fast, and easily implemented.

\section{References:}

[1] M. W. Hirsch and S. Smale, "Differential equations, Dynamical system and linear Algebra," Academic, New York, (1974).

[2] J. M.Thompson and H.B. Stewart, " Non-linear dynamics and Chaos," (Wiley, new york, 1986).

[3] J.Guckenheimer and P.J.Holmes, "Non-linear oscillations, Dynamical systems, and Bifurcations of vector fields," NewYork: Springer-verlag, 2nd printing, (1983).

[4]John H. Mathews,"Numerical methods for Mathematics Science, and Engineering," $2^{\text {nd }}$ EditionPrentice-Hall, Inc. (1992).

[5] J.Della Dora, L.Stolovitch, "Normal forms of differential systems", London Mathematical Society, Lecture note Series (193), CAMBRIDGE UNIVERISITY PRESS.

[6] S.Ushiki, "Normal form for singularities of non-linear differential equations," in computing methods in applied science and engineering, VI, R. Glowinski and J. L. Lions, Eds.Amesterdam, the Netherlands: North Holand, (1984).

[7] S.Ushiki, "Normal form for singularities of vector fields," Japan J.Appl.Math.,Vol.pp-1-37, 1984.

[8] L.O.Chua and H.Kokubu, "Normal forms for non-linear vector fields-part 1: Theory and Algorithm," "IEEE Circuits syst.,vol.35,pp 863-880, (1987).

[9] J. Basto-Gon, calves, A.C. Ferreira, "Normal forms and linearization of vector fields with multiple eigenvalues," J. Math. Anal. Appl. 301 (2005), 219-236.

[10] Takens F., Vanderbauwhede A., "Local invariant manifolds and normal forms," Eds Handbook of dynamical systems, vol. 3, North-Holland, (2009).

[11] Michael T. rosestain, James J. collins and carlo J. De Luca, "A practical method for Calculating Largest Lyapunov exponents from small data setes," Neuro muscolar research center and Department of biomedical Engineering, November (1992).

[12] J.C.Sprott, "Numerical calculation of Largest Lyapunov Exponent,"october13, (1998).

[13] K. Ramasubramanian and M. S. Sriram," Acomparative study of computation of Lyapunov spectra with different algorithms," arXiv:chao-dyn/99909029 v1 17 sep (1999).

[14] Fridaus E. Udwadia and Hubertus F. von Bremen, "An efficient and stable approach for computation of characteristic exponents for continuous dynamical system," Applied Mathematics and computation 121(2001)219-259.

[15] D. Guégan, J. Leroux, "Forecasting chaotic systems: The role of local Lyapunov exponents," Chaos, Solutions and Fractals, 41 (2009a) 2401-2404.

[16] D. Guégan, J. Leroux, "Local Lyapunov Exponents: A New Way to Predict Chaotic Systems, forthcoming in Topics on Chaotic Systems," World Scientific, Eds., (2009)b. 
[17]Gavrylyak M.S., Maksimyak A.P. and Maksimyak P.P. , "Correlation method for measuring the largest Lyapunov exponent in optical fields," Ukr. J. Phys. Opt. (2008), V9, №2 pp119127

[18] M. Sano and Y. Sawada," Measurement of the Lyapunov spectrum from a chaotic time series," PHYSICAL REVIEW LETTERS, vol35.No10, Sep(1985).

[19]Brown, R. [1992] "Generalizations of the Chua Equations," International Journal of Bifurcation \& Chaos in Applied Sciences \& Engineering, vol.2, no.4, pp.889-909.

[20]A. Huang, L, Pivka, C. W. Wu and M. Franz, .Chua's equation with cubic nonlinearity,. Int. J.Bifurcation and Chaos, vol. 6, pp. 2175-2222, 1996. 